\title{
Cognitive training and cognitive rehabilitation for persons with mild to moderate dementia of the Alzheimer's or vascular type: a review
}

\author{
Alex Bahar-Fuchs*1, Linda Clare² and Bob Woods²
}

\begin{abstract}
Cognitive impairments, and particularly memory deficits, are a defining feature of the early stages of Alzheimer's disease and vascular dementia. Interventions that target these cognitive deficits and the associated difficulties with activities of daily living are the subject of ever-growing interest. Cognitive training and cognitive rehabilitation are specific forms of non-pharmacological intervention to address cognitive and non-cognitive outcomes. The present review is an abridged version of a Cochrane Review and aims to systematically evaluate the evidence for these forms of intervention in people with mild Alzheimer's disease or vascular dementia. Randomized controlled trials (RCTs), published in English, comparing cognitive rehabilitation or cognitive training interventions with control conditions and reporting relevant outcomes for the person with dementia or the family caregiver (or both), were considered for inclusion. Eleven RCTs reporting cognitive training interventions were included in the review. A large number of measures were used in the different studies, and meta-analysis could be conducted for several primary and secondary outcomes of interest. Several outcomes were not measured in any of the studies. Overall estimates of the treatment effect were calculated by using a fixed-effects model, and statistical heterogeneity was measured by using a standard chi-squared statistic. One RCT of cognitive rehabilitation was identified, allowing the examination of effect sizes, but no meta-analysis could be conducted. Cognitive training was not associated with positive or negative effects in relation to any of the reported outcomes. The overall quality of the trials was low to moderate. The single RCT of cognitive rehabilitation found promising results in relation to some patient and caregiver outcomes and was generally of high quality. The available evidence regarding cognitive training remains limited, and the quality of the evidence needs to improve. However, there is still no indication of any significant benefits from cognitive training. Trial reports indicate that some gains resulting from intervention may not be captured adequately by available standardized outcome measures. The results of the single RCT of cognitive rehabilitation show promise but are preliminary in nature. Further well-designed studies of cognitive training and cognitive rehabilitation are required to provide more definitive evidence. Researchers should describe and classify their interventions appropriately by using the available terminology.
\end{abstract}

\section{Introduction}

Cognitive impairment is a defining feature of dementia caused by neurodegenerative conditions such as Alzheimer's disease (AD) and cerebrovascular disease. In the milder stages of dementia, cognitive impairments are often the most disabling and distressing features for the individual and their family. For the person with dementia, memory

*Correspondence: alex.baharfuchs@anu.edu.au

${ }^{1}$ Centre for Research on Ageing, Health, and Wellbeing, Australian National

University, 62A Eggleston Road, Canberra, Acton 0200, Australia

Full list of author information is available at the end of the article and other cognitive difficulties can have a major impact on levels of confidence and can lead to anxiety, depression, and withdrawal from activities, which in turn can result in 'excess disability' [1]. Family caregivers are also affected because of the practical impact of cognitive problems on everyday life and the strain and frustration that often result. Interventions to assist with aspects of cognitive functioning, such as memory problems, and associated functional limitations are therefore important in the milder stages of dementia as they may allow the person greater independence and can potentially minimize the risk of 'excess disability'. Interventions for people with mild dementia can be pharmacological, nonpharmacological, or both. Within the broader context of 
non-pharmacological interventions for people with mild dementia, there has been a steady increase in interest in the class of interventions generally referred to as cognition-focused interventions, and these form the focus of the present review.

Although extensive efforts to develop disease-modifying treatments continue, consistently disappointing results from drug trials with various agents have led to considerable doubt that disease-modifying treatments can show a positive effect by the time dementia is fully developed [2], and efforts in this direction are increasingly being shifted to the pre-dementia or even the presymptomatic stage. In contrast, non-pharmacological interventions, and particularly cognition-based interventions, are increasingly being recognized as an important adjunct (and, in some cases, alternative) to pharmacological treatments for individuals with dementia and those at risk of dementia. Nevertheless, earlier studies suggested that cognition-based interventions are not appropriate, as they are ineffective and result in frustration and depression for participants and caregivers [3]. With a growing emphasis on early detection and intervention in dementia care, the need for a clear evidence base for cognition-focused interventions is therefore becoming increasingly apparent [4].

\section{Cognition-focused interventions}

Cognition-focused interventions are interventions that directly or indirectly target cognitive functioning as opposed to interventions that focus primarily on behavioral (for example, wandering), emotional (for example, anxiety), or physical (for example, sedentary lifestyle) function. Several types of cognition-based interventions have been described. The potential benefits of reality orientation and of non-specific stimulation of cognitive functioning for people with dementia have long been recognized. These interventions typically involve engaging the person with dementia in a range of general activities and discussions, are commonly conducted in groups, and are aimed at general enhancement of cognitive and social functioning. A recent Cochrane Review that focuses on interventions falling under this category concluded that general cognitive stimulation and reality orientation approaches consistently produce improvements in general cognition and, in some cases, in selfreported quality of life and well-being, primarily for people with mild to moderate dementia [5].

Progress in understanding the operation of memory and related cognitive functions and of the mechanisms underpinning learning has facilitated the development of more specific approaches designed to help maintain or enhance cognitive functioning and well-being for people with $\mathrm{AD}$ or vascular dementia $(\mathrm{VaD})$, most commonly those in the milder stages. These more recent approaches to cognition-based interventions are most commonly referred to as cognitive training (or 'retraining' or 'remediation' or 'brain training') or cognitive rehabilitation. The present review focuses on these two more recent forms of cognition-based interventions. A more detailed review is published and regularly updated in the Cochrane Database of Systematic Reviews (CDSR) [6]. Because the terms cognitive training and cognitive rehabilitation traditionally have been applied somewhat interchangeably in the literature, Clare and colleagues $[7,8]$ have previously offered the following broad definitions and descriptions with the aim of clarifying the nature of these two related but distinct forms of intervention. Cognitive training typically involves guided practice on a set of standardized tasks designed to reflect particular cognitive functions, such as memory, attention, or problemsolving. Tasks may be presented in paper-and-pencil [911] or computerized [12-14] form or may involve analogs of activities of daily living [15-17]. Tailoring of task difficulty on the basis of the individual performance level and adaptive training (that is, adjustment of task difficulty in response to change in performance level) is becoming increasingly available through computerized packages (for example, [18]). One assumption underlying cognitive training is that practice has the potential to improve or at least maintain functioning in the given domain. An additional assumption is that any effects of practice will generalize beyond the immediate training context. Although this latter assumption has not often been supported by the evidence $[19,20]$, some have argued that the failure to produce transferable benefits is related in part to problems with task design [21]. Recently, some have broadened the definition of cognitive training to include strategy training, which involves the instruction and practice of strategies to minimize cognitive impairment and enhance performance (for example, method of loci and visual imagery) and cognitive exercise [22]. Cognitive training may be offered through individual $[10,16]$ or group $[23,24]$ sessions or facilitated by family members $[17,25]$ with therapist support. In accordance with the suggestion that cognitive training may enhance the effects of pharmacological therapy [26], some studies have evaluated the efficacy of cognitive training in combination with acetylcholinesterase-inhibiting $[11,16,24]$ or other $[12,27]$ medications. In addition, cognitive training for persons with dementia has sometimes been included as a component of supportive interventions for caregivers [28].

Historically, rehabilitation has been viewed as a process aimed at helping people achieve or maintain an 'optimal level of physical, psychological and social functioning' in the context of specific impairments arising from illness or injury [29], thus facilitating participation in preferred activities and valued social roles [30]. More recent views 
of rehabilitation include a deeper appreciation of the complex interplay between disease and ability to function: a disability may persist even once the disease that triggered it has been eliminated, and likewise disability can be reduced in the face of permanent injury or chronic disease [31]. Cognitive rehabilitation, originally developed mainly through work with younger brain-injured people but equally applicable to progressive conditions, refers to the rehabilitation of people with cognitive impairments. Although the concept continues to evolve, cognitive rehabilitation generally refers to an individualized approach to helping people with cognitive impairments, in which those affected, and their families, work together with health-care professionals to identify personally relevant goals and devise strategies for addressing these [32]. The emphasis is not on enhancing performance on cognitive tasks as such but on improving functioning in the everyday context. Cognitive rehabilitation interventions aim to tackle directly those difficulties considered most relevant by the person with dementia and his or her family members or supporters and target everyday situations in the real-life context. Cognitive rehabilitation approaches tend to be implemented in real-world settings since there is no implicit assumption that changes instituted in one setting would necessarily generalize to another. Goals for intervention are selected collaboratively, and interventions are usually conducted on an individual basis.

Both cognitive training and rehabilitation might be accompanied by psychoeducational activities aimed at facilitating an understanding of cognitive strengths and difficulties and by supportive discussion relating to individual emotional reactions or other needs, and where appropriate, links would be made with other possible sources of support [6] Table 1 summarizes the main differences in the attributes of cognitive training and cognitive rehabilitation.

\section{Therapeutic mechanisms}

Cognition-based interventions for persons with acquired disorders of the central nervous system (including traumatic brain injury, stroke, and neurodegenerative conditions) are driven by knowledge of brain-behavior relationships and mechanisms of injury, disease, and recovery. Historically, such interventions reflected two broad conceptual frameworks to the recovery of function following brain illness or injury: a traditional or restorative approach and a contextualized or compensatory approach [33]. Techniques usually associated with cognitive training - such as the repeated exercise of standardized cognitive tests of increasing difficulty, targeting specific cognitive domains - tend to reflect restorative principles and 'thrive on the lure of neuroplasticity' [34]. Some evidence in support of this comes from a recent functional magnetic resonance imaging (fMRI) study that showed increased memory-related brain activation following cognitive training in several brain regions of individuals at high risk of dementia due to mild cognitive impairment (MCI) [35]. Such increased brain activation may be the result of processes of synaptic growth and repair triggered by repeated practice on standardized tests. On the other hand, techniques usually associated with cognitive rehabilitation, such as using strategies to optimize residual cognitive abilities in impaired domains and making the most of unimpaired cognitive abilities, lend themselves more to compensatory approaches. For example, in relation to memory and learning, it is well established that the processes of memory encoding and consolidation, and the subsystem of declarative memory, tend to be profoundly impaired even in the milder stages of AD [36]. Nevertheless, research has shown that, given appropriate conditions and support and sufficient time, people with dementia still have the ability to learn and retain some information and skills despite their memory difficulties [37-39]. A cognitive rehabilitation approach may focus on helping the person with dementia and their families make the most of residual memory ability (for example, by identifying the best ways of taking in important information [40-43] or carrying out important real-life practical skills [44]). Indeed, several learning principles and techniques (for example, errorless learning and spaced retrieval) have been found to lead to improved rates of learning and memory among patients with mild dementia $[45,46]$. Importantly, it is also well documented that despite the severity of memory difficulties, certain memory systems and processes - such as implicit memory (for example, priming and procedural memory) - are relatively preserved in the milder stages of $\mathrm{AD}$ and $\mathrm{VaD}[47,48]$. This profile suggests that interventions may also aim to build on areas of relative strength reflected in preserved aspects of memory and work with patients on strategies to learn information via less impaired components of the memory system. Finally, cognitive rehabilitation interventions also attempt to assist patients in developing ways to compensate for impairments in those aspects of memory that are significantly affected (such as the use of external memory aids or making environmental changes), so as to minimize the cognitive demand of various activities [49-51]. Cognitive rehabilitation interventions use these and other techniques to enhance or maintain everyday functioning and well-being and reduce excess disability for the person with dementia and to reduce strain for family caregivers.

\section{Objectives}

The primary objective of this review was to evaluate the evidence regarding the effects of cognitive training and 
Table 1. Selected differences between cognitive training and cognitive rehabilitation

\begin{tabular}{lll}
\hline & Cognitive training & Cognitive rehabilitation \\
\hline Target & Impairment & Participation restriction \\
Context & Structured tasks and environments & Real-world setting \\
Focus of intervention & Isolated cognitive abilities and processes & $\begin{array}{l}\text { Groups of cognitive abilities and processes required to } \\
\text { perform everyday tasks }\end{array}$ \\
Format & Individualized or group & Individualized \\
Proposed mechanism of action & Mainly restorative; sometimes combined with & A combination of restorative and compensatory \\
& psychoeducation and strategy training & approaches combined with psychoeducation and \\
soals & Improved or maintained ability in specific & Performance and functioning in relation to \\
& cognitive domains & collaboratively set goals
\end{tabular}

cognitive rehabilitation for people with mild to moderate $\mathrm{AD}$ or $\mathrm{VaD}$ in relation to cognitive and non-cognitive outcomes for the person affected and their caregiver. In addition, we consider the nature and quality of the randomized controlled trial (RCT) evidence that is available on this topic.

\section{Methods}

This review is an abridged version of a regularly maintained and recently updated Cochrane Review on the topic. For a detailed description of the review methodology, readers are referred to the complete version in the CDSR. Central elements of the methodology are summarized below.

\section{Inclusion criteria}

To be considered for inclusion, studies had to be RCTs of cognitive training or cognitive rehabilitation (consistent with the definitions provided above) for individuals with mild to moderate $\mathrm{AD}$ or $\mathrm{VaD}$ and had to be published in English. Interventions could be delivered individually or in groups, with or without the inclusion of family caregivers. At a minimum, studies had to include preand post-intervention assessments using standardized measures.

A range of outcomes were considered, and these were broadly classified into the following:

1. Cognitive and non-cognitive outcomes for the person with dementia (for example, performance on objective measures by cognitive domain, self-rated cognitive functioning, mood, well-being, and quality of life).

2. Outcomes for the primary caregiver (mood, burden of care, quality of life, and so on).

3. Effects of the intervention on the course of dementia (global dementia severity and rates of admission to residential care).

Outcomes of studies of cognitive training and of cognitive rehabilitation were considered separately. In addition, separate comparisons were conducted for outcomes in the short term (that is, the first assessment after intervention) and, where available, the medium (2 to 12 months after intervention) and long (>12 months) term. To contribute to the meta-analysis of a given outcome, studies had to have measured the outcome with at least one standardized measure or questionnaire.

\section{Search methods}

The Cochrane Dementia and Cognitive Improvement Group's Specialized Register ALOIS [52] - was most recently searched on 2 November 2012. ALOIS is maintained by the Trials Search Co-ordinator of the Cochrane Dementia and Cognitive Improvement Group and contains studies in the areas of dementia prevention, dementia treatment, and cognitive enhancement in healthy individuals. For a comprehensive list of sources included in the ALOIS database, readers are referred to the ALOIS website [52].

\section{Data collection and analysis}

The latest search results (covering the period of April 2006 to November 2012) were reviewed by AB-F, who identified all relevant RCTs of cognition-based interventions in mild $\mathrm{AD}$ or $\mathrm{VaD}$ and retrieved the full texts. Two review authors (AB-F and LC) independently reviewed each article to determine whether inclusion criteria were met. There were no disagreements regarding the inclusion of studies. All relevant data were extracted from the studies selected for inclusion, recorded in a data-entry form, and entered into Review Manager (RevMan) for statistical analyses. Additional information was sought from study authors as appropriate. Change from baseline statistics was calculated from the group means and standard deviations at baseline, post-intervention, and follow-up. The meta-analysis was conducted on change-from-baseline scores. Outcome measures were treated as continuous measures. In some cases, outcomes were derived from ordinal rating scales; provided that these contained a reasonably large number of categories $(>10)$, the data were treated as continuous variables arising from a normal distribution. 
The mean difference with 95\% confidence intervals was used whenever studies used the same outcome measure, and the standardized mean difference, which is the absolute mean difference divided by the pooled standard deviation, was used when the same outcome was assessed using different measures. Overall estimates of the treatment effect were calculated with a fixed-effects model by using the inverse variance method.

Statistical heterogeneity was assessed by using a standard chi-squared statistic and associated 12 statistic. Consistent with recommendations, heterogeneity was deemed to be present when the chi-squared statistic was significant at a $P$ value of 0.1 or the 12 suggested that more than $40 \%$ of the variability in effect estimate was due to heterogeneity [53]. As no evidence of statistical heterogeneity was found, all analyses were conducted by using a fixed-effects model.

\section{Results}

The electronic searches retrieved a combined total of 1,339 results. After preliminary screening and removal of duplicate studies, 495 records were forwarded to the review authors for further evaluation. Subsequent to title and abstract review by one review author (AB-F), 49 records were selected for closer assessment, and full records were retrieved and reviewed independently by two review authors (AB-F and LC). After review and discussion, three trials that met the inclusion criteria were identified: two trials describing a cognitive training intervention $[14,17]$ and one trial describing a cognitive rehabilitation intervention [54]. The two cognitive training studies were added to the nine studies that were included in earlier versions of the Cochrane Review [9,10,16,23-25,55-57], bringing the total number of studies in the meta-analysis to 11 . Because there were no previous trials of individualized cognitive rehabilitation, no meta-analysis of cognitive rehabilitation could be performed. The flow of studies through the review process is shown in Figure 1.

Significant diversity was noted among the 12 studies on a range of parameters. Seven studies included only participants with a diagnosis of $\mathrm{AD}$, and four of the others included participants with a diagnosis of $\mathrm{AD}, \mathrm{VaD}$, or mixed dementia. In one study [25], participants were included if they had a diagnosis of dementia due to Parkinson's disease, AD, VaD, or mixed dementia, but it was not possible to ascertain how many of the included participants had Parkinson's disease, as data were reported for all etiologies together. In the included studies, severity of dementia varied from very mild to moderate, and this was generally determined on the basis of scores on a measure of dementia severity or global cognition (for example, Clinical Dementia Rating and Mini-Mental State Examination). It appears, but was not

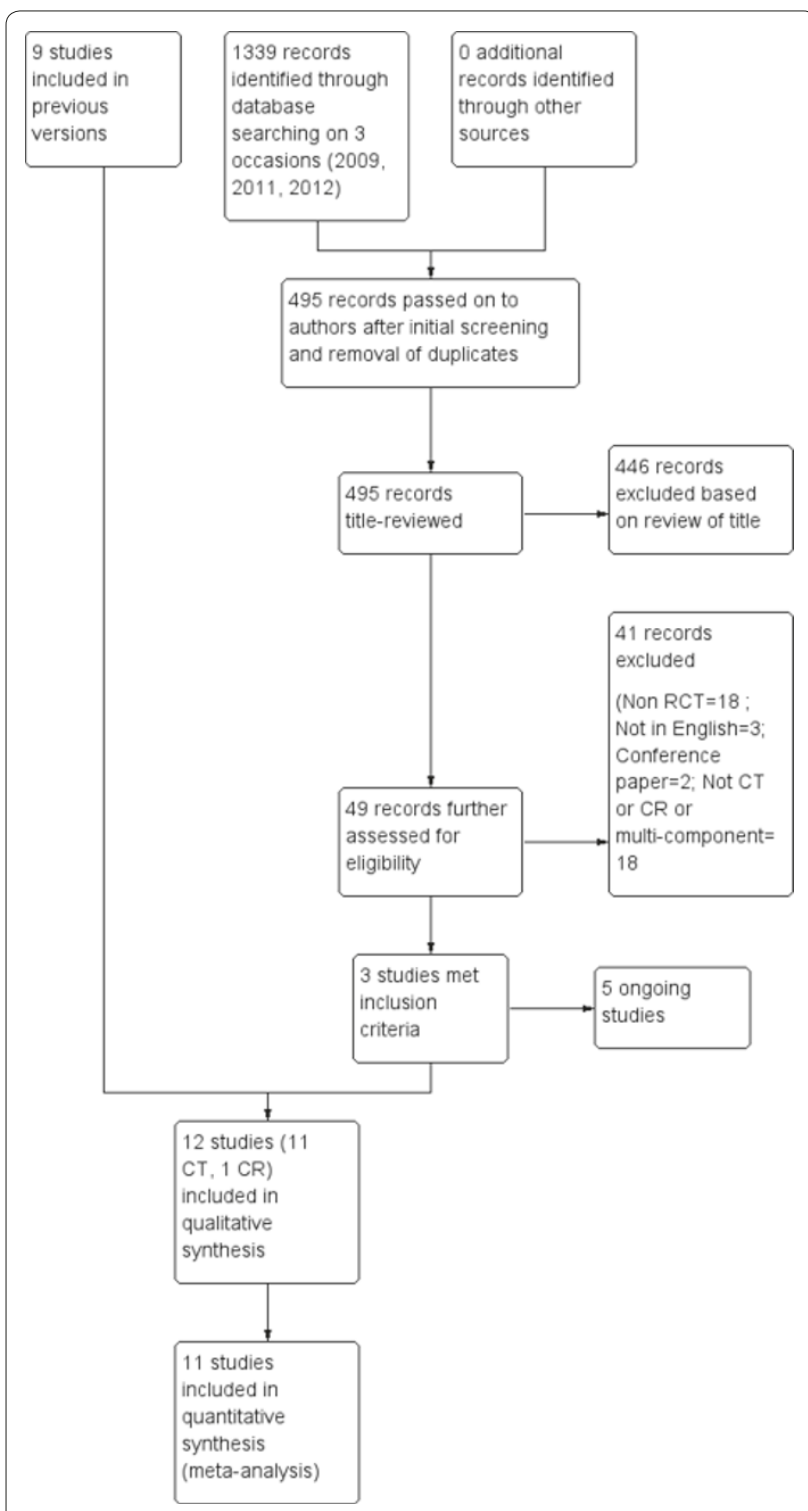

Figure 1. Study flow diagram. $C R$, cognitive rehabilitation; $C T$, cognitive training; $R C T$, randomized controlled trial.

stated explicitly in most studies, that in most cases participants were recruited from the community, although in a small number of studies, participants who resided in residential care homes were also included. The duration of the interventions in the included studies varied considerably, from 4 to 24 weeks. Four studies reported follow-up assessments, and these occurred at 8 weeks, as well as at 3, 6, and 9 months, after the end of treatment. The content of the interventions also varied considerably, from training in the use of compensatory strategies to practice on computerized tasks and to working toward collaboratively derived goals. Selected studies compared two to five conditions, including other 
intervention formats and various control conditions (standard care, wait-list control, and active control). Where more than one comparator intervention was included in a study, the group that was most similar to that included in other studies was selected for analyses. This was usually a 'standard care' group. Selected features of the included studies are summarized in Table 2. Risk of bias in each study was assessed by AB-F, using the Cochrane Collaboration's Risk of Bias tool [53], and subsequently reviewed by LC. Risk of bias was assessed in the following domains: sequence generation, allocation concealment, blinding of participants and investigators, incomplete outcome data, and selective reporting of outcomes. Studies were rated as 'low risk', 'high risk', or 'unclear risk' in each of these domains, according to the criteria specified in the Cochrane Risk of Bias tool. Risk of bias of the various types across studies is summarized in Figure 2. The included studies reported a total of 117 measures (100 measuring patient outcomes and 17 measuring caregiver outcomes) to examine the 22 primary and secondary outcomes selected for examination in this review. For cognitive training interventions, data for meta-analysis were available for eight of the 14 primary outcomes and six of the eight secondary outcomes in the short term. Meta-analysis could be performed on two of the 14 primary outcome measures and two of the eight secondary outcome measures in the medium term. No cognitive training studies reported an outcome measure in the long term. As only one study of cognitive rehabilitation met inclusion criteria for this review, no meta-analysis of cognitive rehabilitation could be conducted.

\section{Cognitive training}

The meta-analysis revealed no differences between cognitive training and control conditions on any of the primary or secondary outcomes included in the analyses. The great majority of comparisons were conducted by using the standardized mean difference method because of the heterogeneity of measures used for each outcome. Depending on the specific analysis, standardized mean differences between cognitive training and control conditions ranged from -0.11 to 0.31 . Furthermore, the evidence from cognitive training interventions to date was generally judged to be of low to moderate quality. Longer-term outcomes related to the trajectory of dementia (that is, severity of dementia and rates of admission to residential care) were not assessed in any of the included studies.

\section{Cognitive rehabilitation}

Because only a single trial of cognitive rehabilitation [54] met criteria for inclusion in the review, no meta-analysis could be conducted. Clare and colleagues [54] found that cognitive rehabilitation was superior to the control condition in relation to a number of outcomes in the short term (patient-reported improvement in goal performance and satisfaction immediately after intervention) and in the medium term (greater satisfaction with memory performance 6 months after intervention). There was also evidence that caregivers of participants in the cognitive rehabilitation group had improved social relationships following the intervention relative to the control condition. Finally, a subset of participants showed changes in task-related brain activation on fMRI following cognitive rehabilitation [58]. This trial was judged to be at relatively low risk of bias in all domains considered and hence to be of generally high quality.

\section{Discussion}

\section{Summary of main results}

The aim of this updated review was to evaluate the current evidence regarding the efficacy of cognitive training and cognitive rehabilitation interventions for people with mild $\mathrm{AD}$ or $\mathrm{VaD}$. Eleven studies of cognitive training were identified for inclusion in the review (and nine of these were included in the previous version of this review), and meta-analysis was performed on several primary and secondary outcomes in the short and medium term. No positive or adverse effects of cognitive training were detected in the meta-analysis. The finding of no adverse effects of cognitive training is relevant in light of proposals from previous commentators (for example, [3]) that cognitive training may have a negative impact, particularly on mood. Only one RCT of individualized cognitive rehabilitation was identified. Hence, no meta-analysis could be conducted.

\section{Overall completeness and applicability of evidence Number of publications meeting inclusion criteria}

Since the publication of the previous version of the Cochrane Review, only two additional RCTs that investigated cognitive training in patients with $\mathrm{AD}$ or $\mathrm{VaD}$ and that met the review criteria were published $[14,17]$. In addition, only a single study met our inclusion criteria for individualized cognitive rehabilitation. Several factors appear to account for the small number of new studies that met criteria for the present review. First, insufficient methodological quality, namely non-randomized trials, led to the exclusion of several published trials (for example, $[59,60])$ from the review. Second, several RCTs of cognition-based interventions either did not meet our definitions of cognitive training and cognitive rehabilitation or described multi-component interventions (for example, $[61,62])$. Issues related to the inclusion criteria used in the present review are further discussed below. A third factor that may have contributed to the appearance of a smaller number of relevant studies in the literature is 


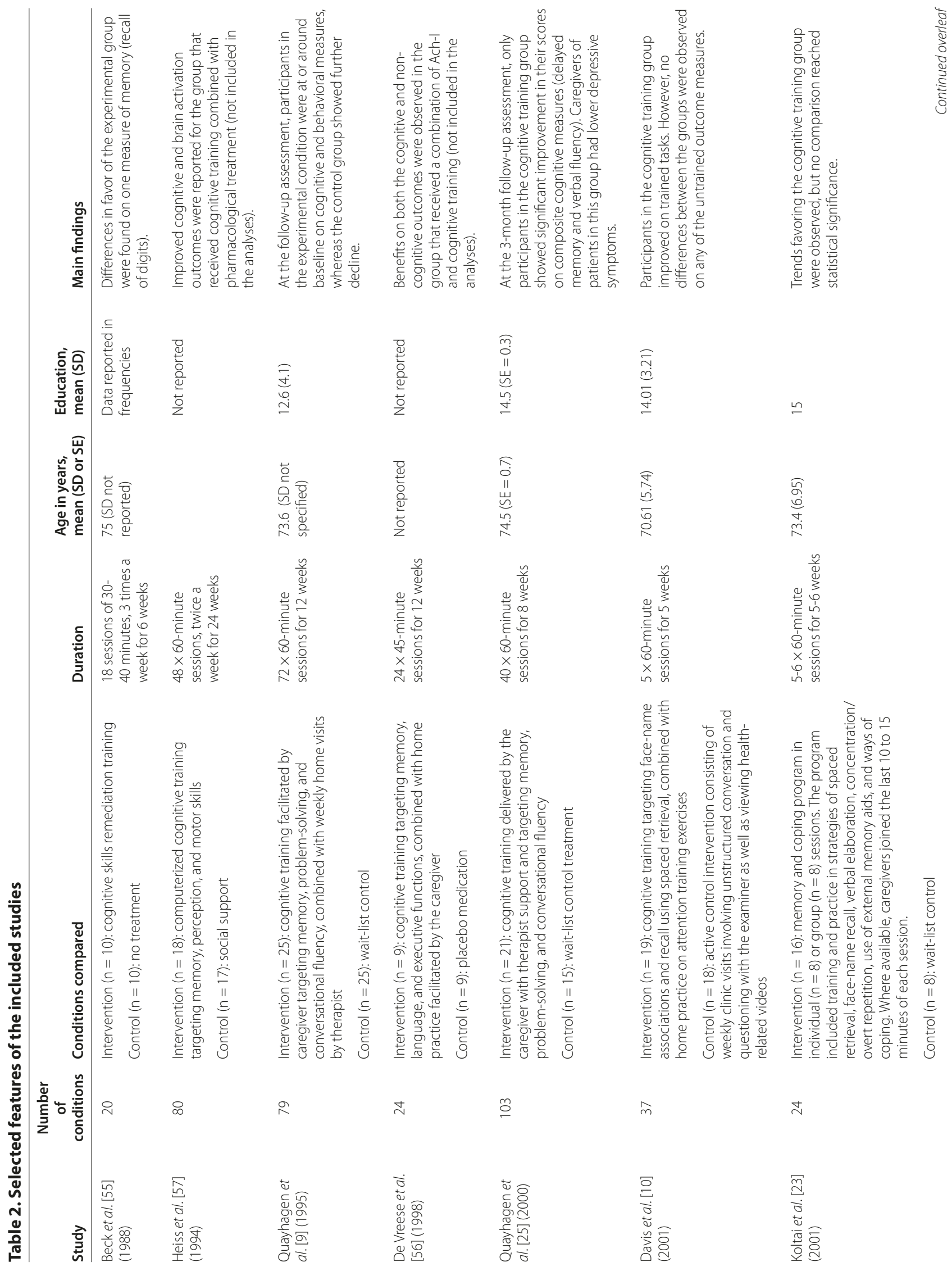




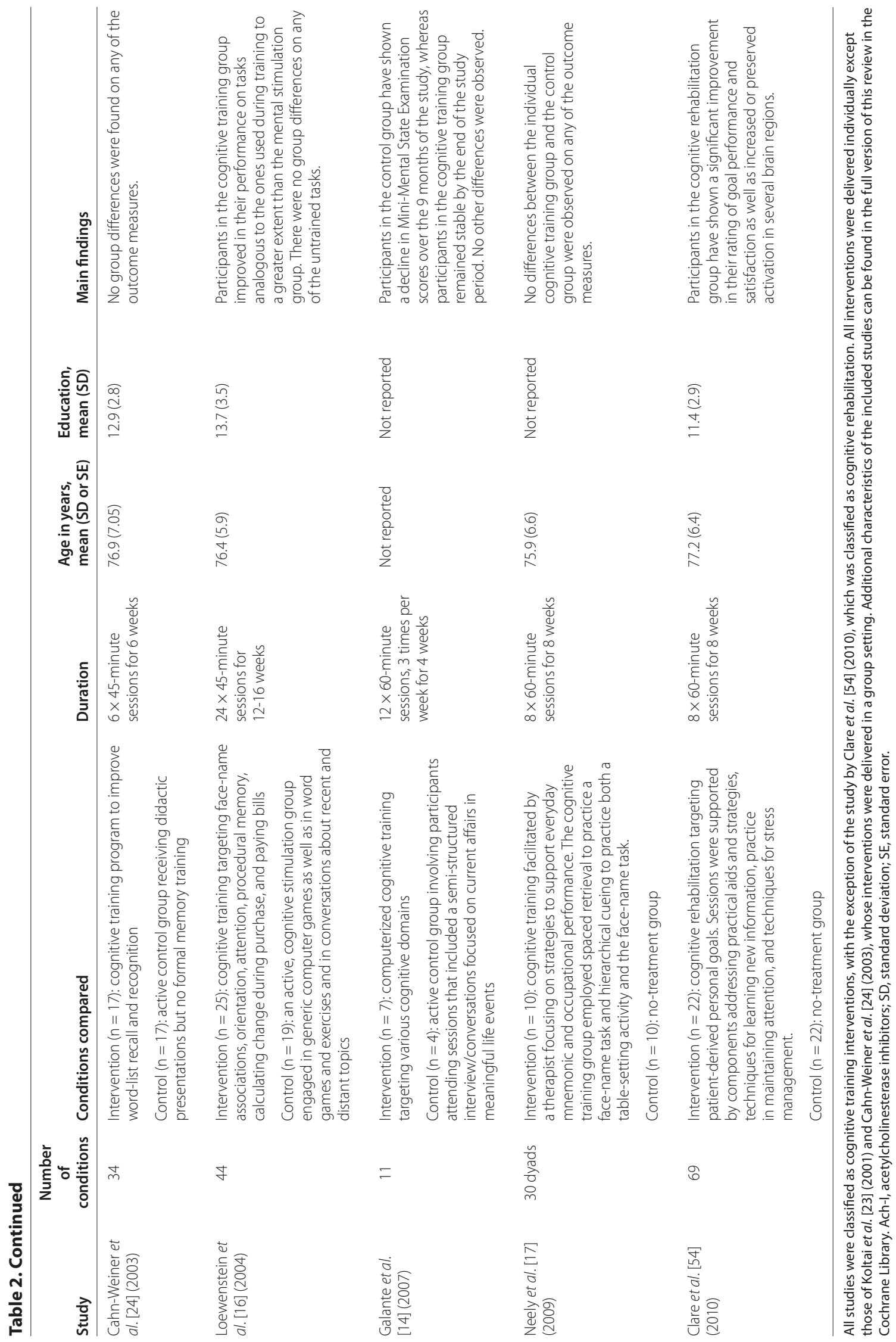




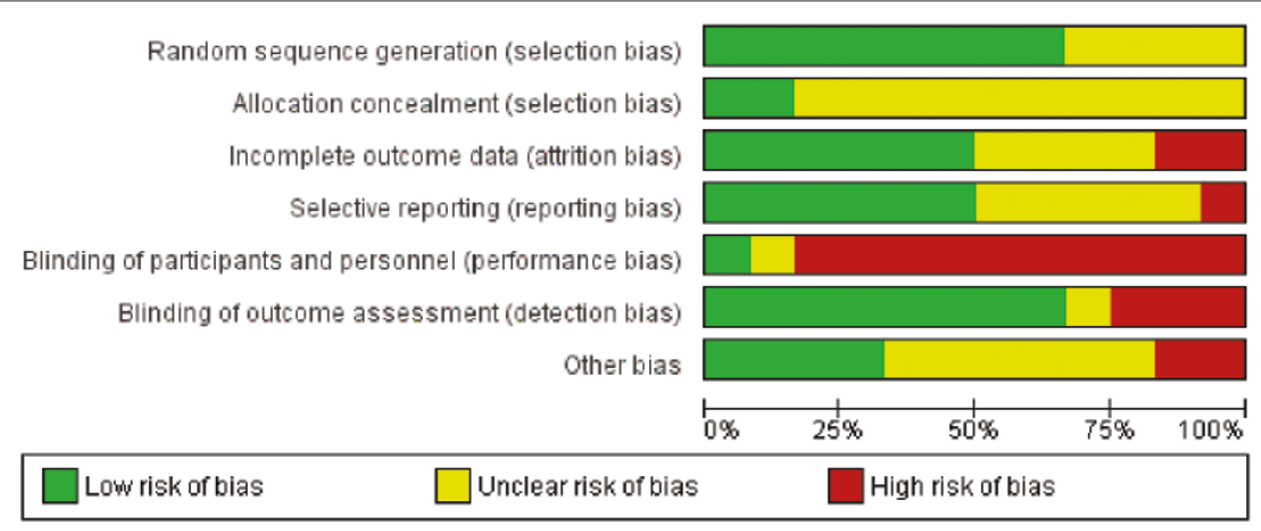

Figure 2. Percentage of studies rated to be of high, low, or unclear risk of bias in each domain.

associated with the widely held belief that interventions pharmacological and non-pharmacological alike - have the greatest chance of success when applied in the earliest possible stage of $\mathrm{AD}$ or $\mathrm{VaD}$. Hence, in recent years, studies have increasingly targeted individuals who do not meet criteria for dementia but who nevertheless show significant cognitive decline - such as persons with amnestic mild cognitive impairment [63,64]. Indeed, many of the records that were retrieved in the updated literature search now focus exclusively on individuals with $\mathrm{MCI}$, and separate reviews focusing on individuals with MCI have been published $[65,66]$.

\section{Issues related to the inclusion of randomized controlled trials only}

The original protocol of the Cochrane Review on which this article is based [6] stated that only RCTs would be included in the review. RCTs have long been regarded as the highest form of evidence in medical research because of the lower risk of bias associated with them. However, most of the studies of cognitive training included in the present review were rated as having substantial risk of bias in several domains, and the quality of evidence was low to moderate. Hence, although the more recent studies are generally of a higher methodological quality and this trend is likely to continue, it might be justifiable, under strict conditions, to include high-quality nonrandomized trials in future reviews to increase the evidence base from which conclusions can be drawn. There are several possible advantages of including highquality non-randomized trials in a systematic review, and the pooled estimates of effect sizes from randomized and non-randomized trials can be analyzed separately [67].

\section{Issues related to definitions of interventions and multi-component interventions}

Despite some progress in the application of a clearer and more consistent terminology to refer to various cognition-based interventions in mild dementia, interventions often continue to be inaccurately labeled. Specifically, studies continue to be published in which interventions are described as cognitive training or as cognitive rehabilitation while they in fact appear to more closely reflect cognitive stimulation or reality orientation (for example, [68]). This state of affairs means that it was generally insufficient, in reviewing the available literature and choosing studies to include in the review, to examine the title used in the publication, and in many cases, the Methods section of a published trial had to be closely scrutinized to clarify whether the actual intervention was consistent with the one suggested by the title.

In addition, the present review excluded trials in which an intervention was described as a combination of elements from various approaches - such as cognitive behavior therapy combined with elements of cognitive rehabilitation (for example, [61]). This decision is related to the fact that different techniques are likely to have different mechanisms of action and that it is generally not possible in such interventions to isolate the contribution of different components to the measured outcomes. The definitions of cognition-based interventions provided in this review essentially reflect groups of intervention techniques that tend to go together, but there is also some overlap in the techniques used in cognitive stimulation, training, and rehabilitation (for example, psychoeducation may be a component of each of these approaches). Because each of these broad approaches to intervention is likely to involve the use of more than one intervention technique with different mechanisms of action (for example, setting goals, learning effective methods to learn new information, and repeated practice), these approaches can also be regarded as essentially 'multicomponent' interventions. Additional work is required in order to better characterize the essential or core components of each of the broad approaches to intervention. It is possible that the inclusion of studies on the basis of 
their use of discrete intervention techniques (for example, goal-setting, practice of structured tasks, and use of specific learning strategies such as errorless learning), rather than whether they fit neatly into the definitions offered here, will prove to be more informative.

\section{Outcomes measured in included studies}

A further issue impacting on the completeness and applicability of the evidence is the range of outcome measures reported in the included studies. Trials, particularly of cognitive training, have traditionally measured mainly cognitive outcomes in the form of performance on standardized cognitive measures. Very few studies, however, have measured non-cognitive outcomes for the person with dementia or the primary caregiver (for example, mood, quality of life, general health, and wellbeing) or outcomes that are likely to be of critical importance to policy-makers - such as outcomes related to the course of dementia (for example, dementia severity and rates of admission to residential care). Although there are obvious methodological constraints to the measurement of some outcomes (for example, long-term outcomes such as admission to residential care), it is nonetheless important that future trials of cognition-based interventions routinely measure and report outcomes other than direct cognitive ones. Given the nature and aims of individualized cognitive rehabilitation interventions, these tend to emphasize individualized goals and activities of daily living over performance on standardized cognitive tests. Indeed, the single trial of cognitive rehabilitation included in the present review measured and reported several important outcomes other than cognitive outcomes that are of direct clinical relevance.

\section{Methodological limitations of included studies}

The lack of significant effects from cognitive training studies must be interpreted in the context of methodological limitations that may have constrained the possibility of demonstrating some significant gains, including issues related to power, choice of control condition, choice of outcome measures, and the impact of individual characteristics that may moderate treatment response.

\section{Power to detect effects}

Many of the included trials are likely to have suffered from limited statistical power to detect effects. Lack of power of individual studies to detect effects is commonly associated with small sample size, which is a frequent limitation in cognition-based interventions for people with mild $\mathrm{AD}$ or $\mathrm{VaD}$. This explanation, however, is unlikely to account for the lack of significant findings, as a meta-analysis is designed to overcome limitations from individual studies associated with such factors as sample size. Indeed, not only was the size of the effects in individual studies small, but - possibly of greater relevance here - the direction of effects associated with some outcomes did not consistently favor cognitive training over the control condition. For example, in three out of the five studies that reported the impact of cognitive training on a global measure of cognition in the short term, the direction of the effect was in favor of the control group, whereas in only one of the trials was the effect clearly in favor of the cognitive training condition. Indeed, such inconsistency in the direction of effects was the case for a substantial number of outcomes reported in the studies, even when the same measures were used by different studies to measure a given outcome. Other possible factors contributing to the difficulty in detecting significant effects might be difficulties in determining the right 'dose' of an intervention (that is, frequency, intensity, and duration of interventions), the presence of 'ceiling' or 'floor' effects rendering it impossible to demonstrate improvements in a given domain, or baseline differences between treatment and control groups.

\section{Choice of control condition}

The difficulty of defining what constitutes an appropriate comparison condition is particularly important since in some studies (for example, [16,24]) cognitive training may have been compared with other active treatments, thus masking potentially beneficial effects. Clinical practice requires the ability to distinguish which of a range of possible psychosocial interventions is most likely to be useful for a given individual, and the study designs used here do not allow this question to be addressed.

\section{Use of neuropsychological tests as cognitive outcomes}

The use of neuropsychological tests to measure cognitive outcomes effectively means that what is actually being assessed is transfer of benefits from trained to untrained tasks, rather than the effects of training on trained tasks. However, as discussed in the introduction, there is very limited evidence in support of such transfer from trained to untrained tasks. But when the trained tasks are in some way analogous to daily activities, improvement in such tasks may have direct relevance to daily functioning, but this would be missed if these benefits were not transferred to performance on standardized neuropsychological tests. For example, Davis and colleagues [10] noted improvement on tasks during training, such as recall of personal information and face-name associations, but this was not captured by the neuropsychological measures selected to assess cognitive outcomes. A further problem with the use of standardized neuropsychological tests before and after the intervention to measure cognitive outcomes involves the potential for practice effects which may obscure possible effects of 
specific treatments. Finally, in some studies, more than one neuropsychological test or self-report scale is used to measure the same outcome (for example, executive function or general well-being). This leads to difficulties in meta-analysis with the choice of the most appropriate or relevant test as the measure of the outcome under consideration.

Moderating role of patient characteristics on intervention outcomes There is an increasing recognition that various patient characteristics have the potential to moderate engagement with and response to the intervention, and as more evidence regarding important moderators becomes available, cognition-focused interventions might be better able to accommodate the effects of such moderators [69]. For example, Koltai and colleagues [23] retrospectively classified participants' level of awareness of their own impairments and found that a higher level of awareness was a predictor of a more successful outcome, a finding that has also been demonstrated in a prospective study of cognitive rehabilitation outcome for a small group of people with mild AD [70].

\section{Study context}

Non-pharmacological interventions are more likely to be affected by the study context, including the health-care setting, and cultural and linguistic factors than drug trials are. Given that the studies reviewed took place in a variety of contexts, one cannot exclude the possibility that cognition-based interventions are better suited for some contexts than others.

\section{Quality of the evidence}

As discussed above, the generally low methodological quality of trials continues to limit the ability to evaluate the evidence base. The methodological quality is gradually improving, and this trend is expected to continue. The quality of most of the studies of cognitive training interventions included in the review was often compromised by several risks of bias that were significant in many of the included studies - particularly as a result of insufficient detail regarding the method used to generate a random group allocation sequence, the concealment of this sequence from relevant members of the research team, and attempts to blind participants or researchers (or both) to group allocation. Hence, the finding of no significant benefits (or harm) from cognitive training interventions needs to be interpreted with caution, and estimates of effect sizes may vary in the future as the evidence comes from studies of better quality. There was only a single study of individualized cognitive rehabilitation, but given the relatively high quality of this trial, cautiously positive (albeit tentative) conclusions regarding the efficacy of this approach can be drawn.

\section{Agreements and disagreements with other studies or reviews}

In recent years, two main systematic reviews that included an examination of the efficacy of cognitive training for people with mild dementia have been published. In reviewing the literature to 2004, Sitzer and colleagues [71] concluded that 'cognitive training evidenced promise in the treatment of $\mathrm{AD}$, with primarily medium effect sizes for learning, memory, executive functions, activities of daily living, general cognitive problems, depression, and self-rated general functioning.' A closer examination of the methodology described in their review reveals important differences that explain the differences with the results of the present review. First, Sitzer and colleagues applied much less strict inclusion criteria and included both randomized and nonrandomized trials (total of 19) as well as studies that included patients with moderate to severe AD. Second, Sitzer and colleagues described their review as one of cognitive training, but of the 14 RCTs that met their inclusion criteria, six were in fact studies of other cognition-based interventions (primarily reality orientation/cognitive stimulation) or multi-component interventions. Indeed, in separate analyses, performed on only the five 'high-quality' trials (all of which were included in the present review), the observed effects were very small and non-significant. It is quite plausible that if studies of cognitive stimulation, training, and rehabilitation for people with mild $\mathrm{AD}$ or $\mathrm{VaD}$ had been assessed together, some benefits would have been detected. However, as there are important differences between the different cognition-focused approaches to intervention, these should be treated separately. Indeed, although the present review did not observe any benefits associated with cognitive training, the results of a single high-quality trial of cognitive rehabilitation tentatively suggest that this approach may be associated with important benefits for the person with dementia and their primary caregiver. In addition, a separate Cochrane Review of cognitive stimulation for mild $\mathrm{AD}$ recently confirmed that this approach was associated with several positive outcomes for the person with dementia [5].

More recently, Olazarán and colleagues [72] reviewed the general literature on the efficacy of 26 categories of non-pharmacological interventions for people with dementia. In relation to cognitive training, these authors concluded that a grade $B$ recommendation (recommendation associated with low-quality RCTs) can be given for the efficacy of individual and group cognitive training for improving cognitive functions. Although this conclusion also seems to be different from the results of the present review, there are important differences between this review and that by Olazarán and colleagues. Specifically, Olazarán and colleagues included in their review participants with any kind of dementia and in fact 
allowed a small proportion of participants with cognitive decline but without confirmed dementia to be included. In addition, these authors did not examine separate cognitive domains, but instead analyzed all cognitive outcomes together, and this was not the approach taken in the present review. These methodological differences most likely account for the differences between the present review and the one by Olazarán and colleagues.

\section{Implications for research}

Cognitive training

Further well-designed single-blind RCTs of cognitive training would help to provide more definitive evidence regarding efficacy. Future research would benefit from consideration of how to capture changes that are currently missed by the available standardized outcome measures, from development of greater consensus in the selection of specific outcome measures, and from identification of the extent to which gains are clinically relevant and generalizable and have the potential to make a difference to the person with dementia and the family caregiver in everyday life. Future research should continue the trend toward devising interventions that include personalized tasks or tasks based on analogs of daily activities. Future research also needs to consider outcomes beyond direct cognitive ones, to describe in greater detail the elements of the intervention used (preferably by using manualized protocols), and to more accurately use existing classifications of cognition-based interventions.

\section{Cognitive rehabilitation}

Additional RCTs of individualized cognitive rehabilitation are needed in order to provide further support for tentatively promising results.

\section{Implications for clinical practice}

\section{Cognitive training}

The review does not provide evidence to support the efficacy of cognitive training. Trial reports indicate that some gains resulting from intervention may not be captured adequately by available standardized outcome measures.

\section{Cognitive rehabilitation}

Data from a single high-quality trial provide preliminary positive results regarding the use of contextualized individual cognitive rehabilitation, emphasizing collaborative goal-setting, to achieve better self-rated competence and satisfaction with personally meaningful activities of daily living. Risk of harm or adverse effects of cognitive rehabilitation is unlikely. The capacity to make firmer treatment recommendations awaits the publication of additional trials of individual cognitive rehabilitation.
This is a version of a Cochrane Review, which is available in the Cochrane Library. Cochrane systematic reviews are regularly updated to include new research and in response to feedback from readers. If you have a comment on this or other Cochrane Reviews of interventions for $\mathrm{AD}$ or VaD, please send it to Sue Marcus, editor of the Cochrane Dementia and Cognitive Improvement Group (sue. marcus@ndm.ox.ac.uk).

\section{Abbreviations}

AD, Alzheimer's disease; CDSR, Cochrane Database of Systematic Reviews: $\mathrm{fMRl}$, functional magnetic resonance imaging; $\mathrm{MCl}$, mild cognitive impairment; $\mathrm{RCT}$, randomized controlled trial; $\mathrm{VaD}$, vascular dementia.

\section{Competing interests}

The authors declare that they have no competing interests.

\section{Acknowledgments}

AB-F is supported by funding from the Dementia Collaborative Research Centre-Early Detection and Prevention and would like to acknowledge the support of the Rosemary Foundation Travel Fellowship awarded to him by the Alzheimer's Australia Research Foundation.

\section{Author details}

'Centre for Research on Ageing, Health, and Wellbeing, Australian National University, 62A Eggleston Road, Canberra, Acton 0200, Australia. 2School of Psychology, Bangor University, Bangor, Gwynedd LL57 2AS, UK.

\section{Published: 7 August 2013}

\section{References}

1. Reifler BV, Larson E: Excess disability in dementia of the Alzheimer's type. In Alzheimer's Disease Treatment and Family Stress: Directions for Research. Edited by Light E, Liebowitz B. New York: Hemisphere; 1990.

2. Salomone S, Caraci F, Leggio GM, Fedotova J, Drago F: New pharmacological strategies for treatment of Alzheimer's disease: focus on disease modifying drugs. Br J Clin Pharmacol 2012, 73:504-517.

3. Small GW, Rabins PV, Barry PP, Buckholtz NS, DeKosky ST, Ferris SH, Finkel SI, Gwyther LP, Khachaturian ZS, Lebowitz BD, McRae TD, Morris JC, Oakley F, Schneider LS, Streim JE, Sunderland T, Teri LA, Tune LE: Diagnosis and treatment of Alzheimer disease and related disorders: consensus statement of the American Association for Geriatric Psychiatry, the Alzheimer's Association and the American Geriatric Society. JAMA 1997, 278:1363-1371.

4. Woods RT, Clare L: Cognition-based therapies and mild cognitive impairment. In Perspectives on Mild Cognitive Impairment. International Perspectives. Edited by Tuokko H, Hultsch D. New York: Taylor \& Francis; 2006:245-264.

5. Woods B, Aguirre E, Spector AE, Orrell M: Cognitive stimulation to improve cognitive functioning in people with dementia. Cochrane Database Syst Rev 2012, 2:CD005562.

6. Clare L, Woods RT, Moniz-Cook ED, Orrell M, Spector A: Cognitive rehabilitation and cognitive training for early-stage Alzheimer's disease and vascular dementia. Cochrane Database Syst Rev 2003, (4):CD003260.

7. Clare L, Woods RT: Cognitive training and cognitive rehabilitation for people with early-stage Alzheimer's disease: a review. Neuropsychol Rehabil 2004, 14:385-401.

8. Clare $\mathrm{L}$ : Cognitive training and cognitive rehabilitation for people with early-stage dementia. Rev Clin Gerontol 2003, 13:75-83.

9. Quayhagen MP, Quayhagen M, Corbeil RR, Roth PA, Rodgers JA: A dyadic remediation program for care recipients with dementia. Nurs Res 1995, 44:153-159.

10. Davis RN, Massman PJ, Doody RS: Cognitive intervention in Alzheimer disease: a randomized placebo-controlled study. Alzheimer Dis Assoc Disord 2001, 15:1-9.

11. de Vreese LP, Neri M, Fioravanti M, Belloi L, Zanetti O: Memory rehabilitation in Alzheimer's disease: a review of progress. Int J Geriatr Psychiatry 2001, 16:794-809.

12. Heiss WD, Kessler J, Slansky I, Mielke R, Szelies B, Herholz K: Activation PET as an instrument to determine therapeutic efficacy in Alzheimer's disease. 
Ann N Y Acad Sci 1993, 695:327-331.

13. Schreiber M, Schweizer A, Lutz K, Kalveram KT, Jäncke L: Potential of an interactive computer-based training in the rehabilitation of dementia: an initial study. Neuropsychol Rehabil 1999, 9:155-167.

14. Galante E, Venturini G, Fiaccadori C: Computer-based cognitive intervention for dementia: preliminary results of a randomized clinical trial. GItal Med Lav Ergon 2007, 29:26-32

15. Zanetti O, Zanieri G, di Giovanni G, de Vreese LP, Pezzini A, Metitieri T, Trabucchi M: Effectiveness of procedural memory stimulation in mild Alzheimer's disease patients: a controlled study. Neuropsychol Rehabil 2001, 11:263-272.

16. Loewenstein DA, Acevedo A, Czaja SJ, Duara R: Cognitive rehabilitation of mildly impaired Alzheimer disease patients on cholinesterase inhibitors. Am J Geriatric Psychiatry 2004, 12:395.

17. Neely AS, Vikstrom S, Josephsson S: Collaborative memory intervention in dementia: caregiver participation matters. Neuropsychol Rehabil 2009, 19:696-715.

18. Peretz C, Korczyn AD, Shatil E, Aharonson V, Birnboim S, Giladi N: Computerbased, personalized cognitive training versus classical computer games: a randomized double-blind prospective trial of cognitive stimulation. Neuroepidemiology 2011, 36:91-99.

19. Owen AM, Hampshire A, Grahn JA, Stenton R, Dajani S, Burns AS, Howard RJ, Ballard CG: Putting brain training to the test. Nature 2010, 465:775-778.

20. Papp KV, Walsh SJ, Snyder PJ: Immediate and delayed effects of cognitive interventions in healthy elderly: a review of current literature and future directions. Alzheimers Dement 2009, 5:50-60.

21. Jaeggi SM, Studer-Luethi B, Buschkuehl M, Su YF, Jonides J, Perrig WJ: The relationship between $n$-back performance and matrix reasoning implications for training and transfer. Intelligence 2010, 38:625-635.

22. Gates N, Sachdev P, Singh MF, Valenzuela M: Cognitive and memory training in adults at risk of dementia: a systematic review. BMC Geriatr 2011, 11:55.

23. Koltai DC, Welsh-Bohmer KA, Smechel DE: Influence of anosognosia on treatment outcome among dementia patients. Neuropsychol Rehabil 2001 11:455-475

24. Cahn-Weiner DA, Malloy PF, Rebok GW, Ott BR: Results of a placebocontrolled study of memory training for mildly-impaired Alzheimer's Disease patients. App/ Neuropsychol 2003, 10:215-223.

25. Quayhagen MP, Quayhagen M, Corbeil RR, Hendrix RC, Jackson JE, Snyder L, Bower D: Coping with dementia: evaluation of four nonpharmacologic interventions. Int Psychogeriatr 2000, 12:249-265.

26. Newhouse PA, Potter A, Levin ED: Nicotinic system involvement in Alzheimer's and Parkinson's diseases: implications for therapeutics. Drugs Aging 1997, 11:206-228.

27. Yesavage JA, Westphal J, Rush L: Senile dementia: combined pharmacologic and psychologic treatment. J Am Geriatr Soc 1981, 29:164-171.

28. Brodaty H, Gresham M, Luscombe G: The Prince Henry Hospital dementia caregivers' training programme. Int J Geriatr Psychiatry 1997, 12:183-192.

29. McLellan DL: Functional recovery and the principles of disability medicine. In Clinical Neurology. Edited by Swash M. London: Churchill Livingstone; 1991:768-790.

30. World Health Organization: International Classification of Functioning, Disability and Health (ICF) [http://www.who.int/classifications/icf/en/].

31. Koehler R, Wilhelm E, Shoulson I: Cognitive Rehabilitation Therapy for Traumatic Brain Injury Cognitive Rehabilitation Therapy for Traumatic Brain Injury: Evaluating the Evidence. Washington, DC: The National Academies Press; 2011.

32. Wilson BA: Towards a comprehensive model of cognitive rehabilitation. Neuropsychol Rehabil 2002, 12:97-110.

33. Ylvisaker M, Hanks R, Johnson-Greene D: Perspectives on rehabilitation of individuals with cognitive impairment after brain injury: rationale for reconsideration of theoretical paradigms. J Head Trauma Rehabil 2002 17:191-209

34. Rabipour S, Raz A: Training the brain: Fact and fad in cognitive and behavioral remediation. Brain Cogn 2012, 79:159-179.

35. Belleville S, Clément F, Mellah S, Gilbert B, Fontaine F, Gauthier S: Trainingrelated brain plasticity in subjects at risk of developing Alzheimer's disease. Brain 2011, 134:1623.

36. Christensen H, Kopelman MD, Stanhope N, Lorentz L, Owen P: Rates of forgetting in Alzheimer dementia. Neuropsychologia 1998, 36:547-557.

37. Kopelman MD: Rates of forgetting in Alzheimer-type dementia and Korsakoff's syndrome. Neuropsychologia 1985, 23:623-638.

38. Little AG, Volans PJ, Hemsley DR, Levy R: The retention of new information in senile dementia. Br J Clin Psychol 1986, 25:71-72

39. Bäckman L: Utilizing compensatory task conditions for episodic memory in Alzheimer's disease. Acta Neurologica Scandinavica 1996, suppl 165:109-113.

40. Camp CJ, Bird MJ, Cherry KE: Retrieval strategies as a rehabilitation aid for cognitive loss in pathological aging. In Cognitive Rehabilitation in Old Age. Edited by Hill RD, Backman L, Stigsdotter-Neely A. Oxford: Oxford University Press; 2000.

41. Clare L, Wilson BA, Breen K, Hodges JR: Errorless learning of face-name associations in early Alzheimer's disease. Neurocase 1999, 5:37-46.

42. Clare L, Wilson BA, Carter G, Roth I, Hodges JR: Relearning of face-name associations in early-stage Alzheimer's disease. Neuropsychology 2002, 16:538-547.

43. Anderson J, Arens K, Johnson R, Coppens P: Spaced retrieval vs memory tape therapy in memory rehabilitation for dementia of the Alzheimer's type. Clin Gerontol 2001, 24:123-139.

44. Josephsson S, Backman L, Borell L, Bernspang B, Nygard L, Ronnberg L: Supporting everyday activities in dementia: an intervention study. Int J Geriatr Psychiatry 1993, 8:395-400.

45. Clare L, Wilson BA, Carter G, Breen K, Gosses A, Hodges JR: Intervening with everyday memory problems in dementia of Alzheimer type: an errorless learning approach. J Clin Exp Neuropsychol 2000, 22:132-146.

46. Dunn J, Clare L: Learning face-name associations in early-stage dementia: comparing the effects of errorless learning and effortful processing. Neuropsychol Rehabil 2007, 17:735-754

47. Brandt J, Rich JB: Memory disorders in the dementias. In Handbook of Memory Disorders. Edited by Baddeley AD, Kopelman MD, Wilson BA. Chichester: John Wiley \& Sons Ltd; 1995.

48. Morris RG: The neuropsychology of Alzheimer's disease and related dementias. In Handbook of the Clinical Psychology of Ageing. Edited by Woods R, Clare L. Chichester: John Wiley \& Sons Ltd; 1996.

49. Kurlychek RT: Use of a digital alarm chronograph as a memory aid in early dementia. Clin Gerontol 1983, 1:93-94.

50. Bourgeois MS: Evaluating memory wallets in conversations with persons with dementia. J Speech Hear Res 1992, 35:1344-1357.

51. Bird M: Behavioural difficulties and cued recall of adaptive behaviour in dementia: experimental and clinical evidence. Neuropsychol Rehabil 2001, 11:357-375.

52. ALOIS: a comprehensive register of dementia studies [http://www.medicine.ox.ac.uk/alois/].

53. Higgins JPT, Altman DG, Sterne JAC: Chapter 8: Assessing risk of bias in included studies. In Cochrane Handbook for Systematic Reviews of Interventions Version 5.1.0 (updated March 2011). Edited by Higgins JPT. Oxford, UK: The Cochrane Collaboration; 2011.

54. Clare L, Linden DE, Woods RT, Whitaker R, Evans SJ, Parkinson CH, van Paasschen J, Nelis SM, Hoare Z, Yuen KS, Rugg MD: Goal-oriented cognitive rehabilitation for people with early-stage Alzheimer disease: a singleblind randomized controlled trial of clinical efficacy. Am J Geriatr Psychiatry 2010, 18:928-939

55. Beck C, Heacock $P$, Mercer $S$, Thatcher R: The impact of cognitive skills remediation training on persons with Alzheimer's disease or mixed dementia. J Geriatr Psychiatry 1988, 21:73-88.

56. De Vreese L, Verlato C, Emiliani S, Schioppa S, Belloi L, Salvioli G, Neri M: Effect size of a three-month drug treatment in $A D$ when combined with individual cognitive retraining: preliminary results of a pilot study. Neurobiol Aging 1998, 19:S213

57. Heiss WD, Kessler J, Mielke R, Szelies B: Long-term effects of phosphatidylserine, pyritinol, and cognitive training in Alzheimer's disease: a neuropsychological, EEG, and PET investigation. Dementia 1994 5:88-98.

58. van Paasschen J, Clare L, Yuen KS, Woods RT, Evans SJ, Parkinson CH, Rugg $M D$, Linden DE: Cognitive rehabilitation changes memory-related brain activity in people with Alzheimer disease. Neurorehabil Neural Repair 2013, 27:448-459.

59. Bentwich J, Dobronevsky E, Aichenbaum S, Shorer R, Peretz R, Khaigrekht M, Marton RG, Rabey JM: Beneficial effect of repetitive transcranial magnetic stimulation combined with cognitive training for the treatment of Alzheimer's disease: a proof of concept study. J Neural Transm 2011, 118:463-471.

60. Hwang HR, Choi SH, Yoon DH, Yoon BN, Suh YJ, Lee D, Han IT, Hong CG: The effect of cognitive training in patients with mild cognitive impairment 
and early Alzheimer's disease: a preliminary study. J Clin Neuro/ 2012, 8:190-197.

61. Kurz A, Thöne-Otto A, Cramer B, Egert S, Frölich L, Gertz HJ, KehI V, Wagenpfeil S, Werheid K: CORDIAL: Cognitive rehabilitation and cognitive-behavioral treatment for early dementia in alzheimer disease: a multicenter, randomized, controlled trial. Alzheimer Dis Assoc Disord 2012, 26:246-253.

62. Graff MJ, Vernooij-Dassen MJ, Thijssen M, Dekker J, Hoefnagels WH, Rikkert MG: Community based occupational therapy for patients with dementia and their care givers: randomised controlled trial. BMJ 2006, 333:1196.

63. Albert MS, DeKosky ST, Dickson D, Dubois B, Feldman HH, Fox NC, Gamst A, Holtzman DM, Jagust WJ, Petersen RC, Snyder PJ, Carrillo MC, Thies B, Phelps $\mathrm{CH}$ : The diagnosis of mild cognitive impairment due to Alzheimer's disease: Recommendations from the National Institute on Aging and Alzheimer's Association workgroup. Alzheimers Dement 2011, 7:270-279.

64. Gauthier S, Reisberg B, Zaudig M, Petersen RC, Ritchie K, Broich K, Belleville S, Brodaty H, Bennett D, Chertkow H, Cummings JL, de Leon M, Feldman H, Ganguli M, Hampel H, Scheltens P, Tierney MC, Whitehouse P, Winblad B; International Psychogeriatric Association Expert Conference on mild cognitive impairment: Mild cognitive impairment. Lancet 2006, 367:1262-1270.

65. Jean L, Bergeron MÈ, Thivierge S, Simard M: Cognitive intervention programs for individuals with mild cognitive impairment: systematic review of the literature. Am J Geriatr Psychiatry 2010, 18:281-296.

66. Martin M, Clare L, Altgassen AM, Cameron MH, Zehnder F: Cognition-based interventions for healthy older people and people with mild cognitive impairment. Cochrane Database Syst Rev 2011, (1):CD006220.

67. Reeves BC, Deeks JJ, Higgins JPT, Wells GA: Chapter 13: Including nonrandomized studies. In Cochrane Handbook for Systematic Reviews of
Interventions Version 510 (updated March 2011). Edited by Higgins JPT. Oxford, UK: The Cochrane Collaboration; 2011.

68. Giordano M, Dominguez LJ, Vitrano T, Curatolo M, Ferlisi A, Di Prima A, Belvedere M, Barbagallo M: Combination of intensive cognitive rehabilitation and donepezil therapy in Alzheimer's disease (AD). Arch Gerontol Geriatr 2010:245-249.

69. Choi J, Twamley EW: Cognitive rehabilitation therapies for Alzheimer's disease: a review of methods to improve treatment engagement and self-efficacy. Neuropsychol Rev 2013, 23:48-62

70. Clare L, Wilson BA, Carter G, Roth I, Hodges JR: Awareness in early-stage Alzheimer's disease: relationship to outcome of cognitive rehabilitation. J Clin Exp Neuropsychol 2004, 26:215-226.

71. Sitzer D, Twamley E, Jeste D: Cognitive training in Alzheimer's disease: a meta-analysis of the literature. Acta Psychiatr Scand 2006, 114:75-90.

72. Olazarán J, Reisberg B, Clare L, Cruz I, Peña-Casanova J, Del Ser T, Woods B, Beck C, Auer S, Lai C, Spector A, Fazio S, Bond J, Kivipelto M, Brodaty H, Rojo JM, Collins H, Teri L, Mittelman M, Orrell M, Feldman HH, Muñiz R: Nonpharmacological therapies in Alzheimer's disease: a systematic review of efficacy. Dement Geriatr Cogn Disord 2010, 30:161-178.

\section{doi:10.1186/alzrt189}

Cite this article as: Bahar-Fuchs A, et al.: Cognitive training and cognitive rehabilitation for persons with mild to moderate dementia of the Alzheimer's or vascular type: a review. Alzheimer's Research \& Therapy 2013 5:35. 